\title{
HENSTOCK INTEGRABLE FUNCTIONS ARE LEBESGUE INTEGRABLE ON A PORTION
}

\author{
ZOLTÁN BUCZOLICH
}

(Communicated by R. Daniel Mauldin)

\begin{abstract}
If a real function $f$ defined on an interval $I \subset \mathbf{R}^{m}$ is Henstock integrable, then one can always find a nondegenerate subinterval $J \subset I$ on which $f$ is Lebesgue integrable.
\end{abstract}

\section{INTRODUCTION}

In his memoir [O; 4.6.4, p. 87] Krzysztof M. Ostaszewski mentioned that "the question posed by Karták in [K]-whether, for a Perron-integrable function, one can find a nondegenerate interval on which it is Lebesgue-integrable-remains unanswered." In this paper we give an affirmative answer to this question. The one-dimensional case of our theorem is an old and well-known result [S, Theorem 1.4, Chapter VIII].

Recall that the (classical) Henstock integral is equivalent to the Perron integral [O, Chapter 2, p. 20]. Since our proof is based on the Henstock integration process we shall use the name Henstock integral in the statement of our theorem.

\section{Preliminaries}

By $\mathbf{R}^{m}$ we denote the $m$-dimensional Euclidean space. By an interval $I$ we mean a set of the form $\times_{i=1}^{m}\left[a_{i}, b_{i}\right]$. The $m$-dimensional Lebesgue measure of the set $E \subset \mathbf{R}^{m}$ is denoted by $|E|$. The Lebesgue integral of a function $f$ over a set $E$ is denoted by $(L) \int_{E} f$. We say that the intervals $I$ and $J$ are non-overlapping if $\operatorname{int}(I) \cap \operatorname{int}(J)=\varnothing$. If the intervals $B_{i} \subset I, i=1, \ldots, k$, are non-overlapping and $I=\cup\left\{B_{i}: i=1, \ldots, k\right\}$, then we say that the set $D=\left\{B_{i}: i=1, \ldots, k\right\}$ is a division of $I$. A partition of an interval $I$ is a collection $P=\left\{\left(B_{i}, x_{i}\right): i=1, \ldots, k\right\}$, where $D(P)=\left\{B_{i}: i=1, \ldots, k\right\}$ is a division of $I$ and $x_{i} \in B_{i}, i=1, \ldots, k$. If a function $\delta: I \rightarrow(0,+\infty)$ and a partition $P$ of $I$ is given we say that $P$ is $\delta$-fine if $B_{i} \subset\left\{y: \operatorname{dist}\left(x_{i}, y\right)<\right.$ $\left.\delta\left(x_{i}\right)\right\}$ for every $i=1, \ldots, k$. If $f$ is a function defined on $I$ and $P$ is a

Received by the editors September 18, 1989.

1980 Mathematics Subject Classification (1985 Revision). Primary 26A39, 26A42.

This paper was prepared while the author visited the University of California at Davis. 
partition of $I$, then we put

$$
\sigma(f, P)=\sum_{i=1}^{k} f\left(x_{i}\right)\left|B_{i}\right| .
$$

We also recall the definition of the Henstock integral.

Definition. A function $f: I \rightarrow \mathbf{R}$ is Henstock integrable on $I$ if there is a real number denoted by $(H) \int_{I} f$ with the following property: for every $\varepsilon>0$, there is a $\delta: I \rightarrow(0,+\infty)$ such that

$$
\left|\sigma(f, P)-(H) \int_{I} f\right|<\varepsilon
$$

for each $\delta$-fine partition of $I$.

We remark that Henstock integrable functions are measurable. This holds even for generalizations of the Henstock integral, see for example [P1, 4.3 Corollary] or [P2, 4.5 Corollary].

THE MAIN RESULT

Theorem. If $I \subset \mathbf{R}^{m}$ is an interval and $f$ is Henstock integrable on $I$, then there exists a nondegenerate interval $J \subset I$ such that $f$ is Lebesgue integrable on $J$.

Proof. Using the existence of $(H) \int_{I} f$, choose $\delta: I \rightarrow(0,+\infty)$ such that if $P$ and $P^{\prime}$ are $\delta$-fine partitions of $I$ then

$$
\left|\sigma(f, P)-\sigma\left(f, P^{\prime}\right)\right|<|I| .
$$

Put $A_{n}=\{x \in I:|f(x)|<n, \delta(x)>1 / n\}$. Since $\delta(x)$ is positive and $f(x)$ is finite we have $\bigcup_{n \in \mathbf{N}} A_{n}=I$, and, by Baire's Category Theorem, there exists an $n \in \mathbf{N}$ and a portion $J \subset I$ such that $A_{n}$ is dense in $J$. We can also assume that $\operatorname{diam}(J)<1 / n$. We shall prove that $(L) \int_{J} f$ exists. For a contradiction suppose that this is not true. Then $(L) \int_{J}|f|=+\infty$, and we can choose a measurable set $E \subset J$ such that

$$
\left|(L) \int_{E} f\right|>3 \cdot n \cdot|I| .
$$

Put

$$
g(x)= \begin{cases}f(x), & \text { if } x \in E, \\ 0, & \text { otherwise. }\end{cases}
$$

Since $g(x)$ is Lebesgue and a fortiori Henstock integrable, we choose $\delta^{\prime}: J \rightarrow$ $(0,+\infty)$ such that $\delta^{\prime}(x)<\delta(x)$ for $x \in J$, and if $P^{\prime}$ is a $\delta^{\prime}$-fine partition of $J$ then

$$
\left|\sigma\left(g, P^{\prime}\right)-(L) \int_{E} f\right|<n|I| .
$$

Suppose that $P^{\prime}$ is a fixed $\delta^{\prime}$-fine partition of $J$. Choose $P^{*}$ a $\delta$-fine partition of $I \backslash J$ and put $P_{1}=P^{\prime} \cup P^{*}$. Then $P_{1}$ is a $\delta$-fine partition of $I$. 
Suppose that $P^{\prime}=\left\{\left(B_{i}, x_{i}\right): i=1, \ldots, k^{\prime}\right\}$. If $x_{i} \notin E$ then put $x_{i}^{\prime \prime}=x_{i}$. If $x_{i} \in E$ then, using the density of $A_{n}$ in $J$, choose an $x_{i}^{\prime \prime} \in A_{n} \cap B_{i}$. Put $P^{\prime \prime}=$ $\left\{\left(B_{i}, x_{i}^{\prime \prime}\right): i=1, \ldots, k^{\prime}\right\}$. Since $\delta(x)>1 / n$ for $x \in A_{n}, \operatorname{diam}(J)<1 / n$, and $\delta^{\prime}<\delta$ the partition $P^{\prime \prime}$ is $\delta$-fine on $J$. Put $P_{2}=P^{\prime \prime} \cup P^{*}$. Then $P_{2}$ is $\delta$-fine on $I$. We obtain

$$
\begin{aligned}
|I|>\left|\sigma\left(f, P_{1}\right)-\sigma\left(f, P_{2}\right)\right|= & \left|\sum_{\left(B_{i} x_{i}\right) \in P^{*}} f\left(x_{i}\right)\right| B_{i}\left|+\sum_{\left(B_{i}, x_{i}\right) \in P^{\prime}} f\left(x_{i}\right)\right| B_{i} \mid \\
& -\sum_{\left(B_{i}, x_{i}\right) \in P^{*}} f\left(x_{i}\right)\left|B_{i}\right|-\sum_{\left(B_{i}, x_{i}^{\prime \prime}\right) \in P^{\prime \prime}} f\left(x_{i}^{\prime \prime}\right)\left|B_{i}\right| \mid \\
& =\left|\sum_{\left(B_{i}, x_{i}\right) \in P^{\prime}} f\left(x_{i}\right)\right| B_{i}\left|-\sum_{\left(B_{i}, x_{i}^{\prime \prime}\right) \in P^{\prime \prime}} f\left(x_{i}^{\prime \prime}\right)\right| B_{i}||=\Psi_{0} .
\end{aligned}
$$

Using the fact that if $x_{i} \notin E$ then $x_{i}=x_{i}^{\prime \prime}$, we have

$$
\begin{aligned}
\Psi_{0} & =\left|\sum_{x_{i} \in E,\left(B_{i}, x_{i}\right) \in P^{\prime}} f\left(x_{i}\right)\right| B_{i}\left|-\sum_{x_{i} \in E,\left(B_{i}, x_{i}^{\prime \prime}\right) \in P^{\prime \prime}} f\left(x_{i}^{\prime \prime}\right)\right| B_{i}|| \\
& >\left|\sigma\left(g, P^{\prime}\right)\right|-\left|\sum_{x_{i} \in E,\left(B_{i}, x_{i}^{\prime \prime}\right) \in P^{\prime \prime}} f\left(x_{i}^{\prime \prime}\right)\right| B_{i}||=\Psi_{1}-\Psi_{2} .
\end{aligned}
$$

Recalling that $\left|(L) \int_{J} g\right|>3 \cdot n \cdot|I|$ and $\left|\sigma\left(g, P^{\prime}\right)-(L) \int_{J} g\right|<n|I|$, we obtain $\Psi_{1}>2 n|I|$. To estimate $\Psi_{2}$ recall that $x_{i}^{\prime \prime} \in A_{n}$ and hence $\left|f\left(x_{i}^{\prime \prime}\right)\right|<n$. Thus $\Psi_{2}<n|J| \leq n|I|$. Therefore we obtain $|I|>\Psi_{1}-\Psi_{2}>2 n|I|-n|I|=n|I| \geq|I|$, a contradiction proving our theorem.

\section{REFERENCES}

[K] K. Karták, K teorii vicerozmèrného integrálu, Časopis Pešt. Mat. 80 (1955), 400-414.

[O] K. M. Ostaszewski, Henstock integration in the plane, Mem. Amer. Math. Soc., no. 63, Amer. Math. Soc., Providence, RI, 1986.

[P1] W. F. Pfeffer, The multidimensional fundamental theorem of calculus, J. Austral. Math. Soc. Ser. A 43 (1987), 143-170.

[P2] - The divergence theorem, Trans. Amer. Math. Soc. 295 (1986), 665-685.

[S] S. Saks, Theory of the integral, Hafner, New York, 1937.

Eötvös Loránd University, Department of Analysis, Budapest, Múzeum krt 6-8, H1088, HUNGARY 